\title{
Inherent Differences between Bound and Radiation Fields
}

\author{
Eliahu Comay \\ Charactell Ltd., Tel-Aviv, Israel \\ Email: elicomay@post.tau.ac.il
}

How to cite this paper: Comay, E. (2018) Inherent Differences between Bound and Radiation Fields. Open Access Library Journal, 5: e4517.

https://doi.org/10.4236/oalib.1104517

Received: March 19, 2018

Accepted: April 22, 2018

Published: April 25, 2018

Copyright $\odot 2018$ by author and Open Access Library Inc.

This work is licensed under the Creative Commons Attribution International License (CC BY 4.0).

http://creativecommons.org/licenses/by/4.0/

\begin{abstract}
The purpose of the paper is to use fundamental theoretical and experimental elements of electrodynamics for deriving properties of radiation fields and of bound fields. A wide variety of examples prove that radiation fields and bound fields do not represent the same physical object. This conclusion is new. Some examples belong to the classical domain and others belong to the quantum domain. Consequences of this outcome affect several physical issues. In particular, these fields should be treated separately. For this reason, changes must be introduced to the present form of the fields' Lagrangian density of quantum electrodynamics, where the fields tensor $F^{\mu \nu}$ is a sum of bound and radiation fields. Since the Lagrangian density is a key element of the theory, its revision may entail changes of other specific issues. The recent failure of quantum electrodynamics to explain the electron and the muon data of the proton charge radius supports this conclusion.
\end{abstract}

\section{Subject Areas}

Theoretical Physics, Electrodynamics

\section{Keywords}

Maxwellian Electrodynamics, Bound Fields, Radiation Fields, Electromagnetic Lagrangian Density, Quantum Electrodynamics

\section{Introduction}

Maxwell has derived his equations of electromagnetic fields from known formulas of radiation-free fields which have been constructed on the basis of experimental data. He has added a term called Maxwell displacement current and derived a set of differential equations which are consistent with the continuity 
equation (see [1], pp. 217-218)

$$
\nabla \cdot \boldsymbol{J}+\frac{\partial \rho}{\partial t}=0
$$

A mathematical analysis of Maxwell equations yields solutions that describe electromagnetic radiation. At that time there was no laboratory confirmation for these waves. Maxwell's electromagnetic radiation shares two important properties with light waves: They are transverse waves that travel at the speed of light in every inertial frame. Later, Hertz has detected electromagnetic radiations in experiment [2]. These waves are now extensively used in theoretical work as well as in modern technology.

This evidence shows that the scientific recognition of bound fields and of radiation fields has a different history. Obviously, this fact says nothing on the problem of whether bound fields and radiation fields represent an identical physical object. This problem is the main topic of the present work. It is studied by an examination of relevant experimental data as well as by an analysis of the theory's mathematical structure. The analysis applies to many specific examples where some of which belong to classical electrodynamics and others belong to the quantum domain.

The variational principle plays a central role in the following discussion. This principle is adopted by many modern textbooks on field theory [3] [4]. For example, it is stated that the variational principle and its Lagrangian function are "the foundation on which virtually all modern theories are predicated" (see [5], p. 353). The results of this work prove that the present form of the Lagrangian density of electrodynamics should be changed. Evidently, due to the primary role of the Lagrangian density in the theoretical structure of electrodynamics, many specific theoretical changes are expected to follow this conclusion.

This work uses standard notation of relativistic expressions and a system of units where $\hbar=c=1$. The metric is diag. $(1,-1,-1,-1)$. The second section defines radiation fields and bound fields. The third section describes a wide variety of examples, each of which shows inherent differences between bound fields and radiation fields. The fourth section discusses some results that are obtained from the physical differences between these fields. The last section contains concluding remarks.

\section{A Definition of Radiation Fields and Bound Fields}

A definition of radiation fields also defines bound fields, because the latter are the fields of a given system of charges that are not radiated. The present form of the Lagrangian density of electromagnetic fields together with the interaction term is (see [1], p. 596; [3], p. 75; [4], p. 349)

$$
\mathcal{L}_{E M}=-\frac{1}{16 \pi} F^{\mu v} F_{\mu v}-e j^{\mu} A_{\mu},
$$

where the electromagnetic field tensor is (see [1], p. 550; [3], p. 65) 


$$
F^{\mu \nu}=g^{\mu \alpha} g^{\nu \beta}\left(A_{\beta, \alpha}-A_{\alpha, \beta}\right)=\left(\begin{array}{cccc}
0 & -E_{x} & -E_{y} & -E_{z} \\
E_{x} & 0 & -B_{z} & B_{y} \\
E_{y} & B_{z} & 0 & -B_{x} \\
E_{z} & -B_{y} & B_{x} & 0
\end{array}\right),
$$

$A_{\mu}$ is the 4-potential of the fields, $e$ denotes the electric charge and $j^{\mu}$ denotes the probability 4 -current.

Let us examine a system of point charges. Actual calculations prove that the fields of a point charge can be derived from the Lienard-Wiechert 4-potential (see [1], p. 656; [3], p. 174)

$$
A_{\mu}=e \frac{v_{\mu}}{R^{\alpha} v_{\alpha}} .
$$

Here $R^{\mu}$ and $v^{\mu}$ denote the charge's retarded coordinates and velocity, respectively. The electromagnetic fields are (see [1], p. 657; [3], p. 175)

$$
\boldsymbol{E}=e \frac{1-v^{2}}{(R-\boldsymbol{R} \cdot \boldsymbol{v})^{3}}(\boldsymbol{R}-\boldsymbol{v} R)+\frac{e}{(R-\boldsymbol{R} \cdot \boldsymbol{v})^{3}} \boldsymbol{R} \times[(\boldsymbol{R}-\boldsymbol{v} R) \times \boldsymbol{a}]
$$

and

$$
\boldsymbol{B}=\boldsymbol{R} \times \boldsymbol{E} / R .
$$

Here $\boldsymbol{R}$ is the 3-vector that is related to the retarded 4-vector $R^{\mu}$, and $\boldsymbol{v}$ and $\boldsymbol{a}$ denote the charge's retarded velocity and acceleration, respectively. The first term of (5) and that of (6) are called velocity fields and the second ones are called acceleration fields. At a large enough distance (called the far zone) velocity fields decrease like $R^{-2}$ whereas acceleration fields decrease like $R^{-1}$. Therefore, velocity fields can be ignored at the far zone.

The fields at the far zone are called radiation fields. They are obtained from the interference of acceleration fields of all charges of the system. These fields decrease like $1 / R$. Thus, the associated Poynting vector (see [1], p. 237, [3], p. 81)

$$
\boldsymbol{S}=\boldsymbol{E} \times \boldsymbol{B} / 4 \pi
$$

decreases like $1 / R^{2}$. This is a necessary condition for the existence of radiation energy which is emitted from the system. And indeed, actual calculations show that at the far zone the fields $E, B$ are perpendicular to each other and to the Poynting vector $S$ (see [3], p. 185). In this caes, the Poynting vector (7) points outwards and represents the current of the radiated energy.

The Poynting vector (7) together with energy conservation of electrodynamics proves the role of velocity fields. Indeed, the product of the fields in (7) shows that effects of velocity fields decrease either like $R^{-4}$ or like $R^{-3}$. In either case, velocity fields do not contribute to energy that flows outwards from the system of charges. Hence, energy conservation proves that velocity fields are pure bound fields and energy which is associated with these fields does not leave the charges' vicinity.

\section{Differences between Radiation Fields and Bound Fields}

Let us examine differences between radiation fields and bound fields that have a 
solid physical basis. For this end, electromagnetic properties of specific experiments are analyzed. Other examples are derived from theoretical arguments. Electrodynamics is a mature science and its theory successfully explains experimental data. The fact that this theory is used by physicists and engineers in their work demonstrates its reliability. Each of the items discussed in this section shows that radiation fields and bound fields are inherently different physical objects.

\subsection{Atomic States and External Fields}

Let us use the hydrogen atom as an experimental device and examine its interaction with external radiation fields and with external bound fields. These issues are regarded here as experimental procedures, because the experimenter controls the external fields. It is well known that quantum mechanics provides a good explanation of the hydrogen atom data. Therefore, results of actual experiments can be replaced by information which is found in quantum mechanical textbooks.

Let us take the $1 s$ ground state of the hydrogen atom whose quantum numbers are $l=0, s=1 / 2, j=1 / 2$. If an incoming photon has the appropriate energy which is consistent with energy conservation then it induces a transition to the $2 p$ excited state whose spatial angular momentum is $l=1$. This is an example of the selection rules of this kind of transition where the difference between the initial angular momentum $l$ and the final angular momentum $l^{\prime}$ and between the corresponding $m$ values are (see [6], p. 264, 265)

$$
l-l^{\prime}= \pm 1 ; m-m^{\prime}=0, \pm 1 .
$$

This process conserves angular momentum and parity. Hence, it is concluded that the spin/parity of the photon is $j^{\pi}=1^{-}$(see the photon data in [7]).

Another kind of the hydrogen atom interaction with external fields is the weak Zeeman effect where the ground state of the hydrogen atom is put in an external rather weak uniform magnetic field (see [6], pp. 292-294; [8], pp. 476-481). Here the ground state splits and the energy of $s_{z}=1 / 2$ is not the same as that of $s_{z}=-1 / 2$. Unlike the photon's case, this energy split increases continuously with the intensity of the external magnetic field. The parity of the magnetic field is even (see [1], p. 249). Therefore, another property of the interaction with an external magnetic field says that this interaction does not alter the parity of the hydrogen atom quantum state. Hence, there is a striking difference between a real photon and a static magnetic field. Indeed, the photon's parity is odd whereas the parity of magnetic field is even.

Parity is a well defined property of systems whose state is determined by strong or electromagnetic interaction. The previous examples show fundamental differences between radiation fields and magnetic bound fields: The parity of a real photon is odd whereas that of a static magnetic field is even.

\subsection{Angular Momentum}

The Zeeman effect shows another aspect of the inherent difference between 
bound magnetic field and radiation. As shown above, the parity of the magnetic field is even. Therefore, its expansion in eigenfunctions of angular momentum contains only even values of the quantum number $l$ (see [6], pp. 73-74; [8], p. 96). By contrast, it is already shown above that the spin of the photon is unity. Hence, angular momentum data prove that a static magnetic field and a photon are inherently different physical objects.

\subsection{Atomic Properties}

The interaction of an atom with external bound fields is discussed in the previous subsections. The following lines describe properties of bound fields associated with the charged particles of a free atom. Angular momentum is an important physical quantity which is used in classical and quantum physics. Evidently, atomic properties belong to the quantum domain (see [6], pp. 141-149; [8], pp. 78-106). These textbooks demonstrate that the angular momentum of an atomic state is derived from an application of the angular momentum operator to the wave function of massive particles of the quantum system. Here the electromagnetic bound fields of the atomic charged particles make no contribution to this quantity.

Parity is another important attribute of a quantum system whose state is determined by strong or by electromagnetic interactions (see [6], p. 139; [8], pp. 94-96). Like the angular momentum case, the parity of an atomic state is derived from an application of the parity operator to the wave function of massive particles of the quantum system. Here the atomic bound fields make no contribution to this quantity.

This treatment of angular momentum and of parity of atomic states has a solid experimental support. By contrast, fields of a real photon are not ignored and the selection rules (8) prove that an interaction with an incoming real photon changes angular momentum and parity of an atomic state.

The following statements are derived from the examples described above: radiation fields are related to a genuine physical entity, which is called a real photon. These fields cannot be ignored in a description of their atomic interaction, because an interaction with a real photon changes the atomic angular momentum and parity. By contrast, bound fields are ignored in calculations of atomic angular momentum and parity. This issue demonstrates another inherent difference between bound fields and radiation fields.

The fact that bound fields are ignored in a quantum calculation of angular momentum and parity means that if bound fields represent a particle then its spin and parity are $j^{\pi}=0^{+}$. This is inconsistent with the spin and parity of a real photon, which are $j^{\pi}=1^{-}$.

\subsection{Kinematical Properties}

Special relativity and Maxwell equations are well established physical theories.

These theories state that in every inertial frame electromagnetic radiation 
propagate at the speed of light.

Let us examine the bound electric field of a system that comprises one charge in the inertial frame where it is at rest. Like the charge itself, the electric field is motionless. Hence, due to the laws of special relativity, there is no inertial frame where this field moves at the speed of light. The same result holds for the bound magnetic field of a superconducting ring that carries an electric current.

These examples show inherent differences between electromagnetic fields of radiation and bound electromagnetic fields.

\subsection{Lorentz Invariants of Fields}

Electromagnetic fields have two Lorentz invariants (see [3], pp. 67, 68)

$$
\text { Inv }_{1}=\frac{1}{2} F_{\mu \nu} F^{\mu v}=B^{2}-E^{2}
$$

and

$$
\operatorname{Inv} v_{2}=\frac{1}{2} \varepsilon_{\mu v \alpha \beta} F^{\mu v} F^{\alpha \beta}=\boldsymbol{E} \cdot \boldsymbol{B}
$$

where $\varepsilon_{\mu v \alpha \beta}$ is the completely antisymmetric unit tensor of the fourth rank.

Let us examine fields of free electromagnetic particles. A real photon is the quantum version of electromagnetic radiation emitted from a specific source. In the units used herein, radiation fields have the following properties (see [3], p. 185)

$$
\begin{gathered}
|\boldsymbol{E}|=|\boldsymbol{B}|, \\
\boldsymbol{E} \perp \boldsymbol{B} .
\end{gathered}
$$

A substitution of (11) into the first fields invariant (9) yields a null value.

Let us compare this result with electromagnetic fields of a single pointlike charge. Evidently, this charge moves inertially because the system does not contain other physical objects with which it can interact. In principle, invariants of fields can be calculated in any inertial frame. Thus, it is simpler to examine the fields at the frame where the charge is at rest. Here one finds that at every spatial point outside that of the charge, the fields are

$$
|\boldsymbol{E}|>0, \boldsymbol{B}=0 .
$$

Contrary to the photon case, a substitution of the charge's fields (13) into the first fields invariant (9) yields a negative value throughout all points of the entire space-time (except at the location of the point-charge).

The following issues describe another aspect of this matter. In the case of bound fields there are devices that produce static magnetic fields in a specific inertial frame. (For example, a motionless ring along which a constant electric current flows produces a static magnetic field.) This example, together with that of a single charge, show time-independent electric and magnetic fields. By contrast, the invariant (9) and the relations (11) and (12) between radiation fields prove that such a separation between the electric and the magnetic fields 
cannot be done for radiation fields.

\subsection{Wigner's Work}

Wigner has analyzed the irreducible representations of the inhomogeneous Lorentz group [4] [9] [10] [11]. Important results of his work state that a massive quantum particle has a well defined mass and spin. A massless particle belongs to a different category. By definition, it has a zero mass and it moves at the speed of light in all inertial frames. Instead of spin, it has two components of helicity.

The constant speed of light in the vacuum is recognized as an inherent property of Maxwellian wave equation of electromagnetic potentials and of their fields (see [3], p. 117). In the standard vector notation, this equation is

$$
\Delta \boldsymbol{A}-\frac{1}{c^{2}} \frac{\partial^{2} \boldsymbol{A}}{\partial t^{2}}=0
$$

where the speed of light $c$ is written explicitly. This equation demonstrates relativistic properties of Maxwellian electrodynamics, where the wave propagate at $c$ in every inertial frame.

A special property of the speed of electromagnetic waves $c$ is that the photon is a massless particle. This fact is consistent with the form of the electromagnetic fields' Lagrangian density (2). Indeed, unlike the case of a Lagrangian density of a massive quantum particle, the Lagrangian density of Maxwellian fields (2) is mass-independent.

The Poynting vector (7) together with the Lorentz invariants (9) and (10) prove an important property of radiation fields: They carry a nonvanishing amount of linear momentum density in every inertial frame. Simple mechanical arguments show that a nonvanishing linear momentum is a necessary condition for the existence of a moving elementary particle. In particular, this property is a requirement for a consistent definition of a real photon.

Let us examine the fields of a system that comprises a single charged particle in the inertial frame where it is motionless. In this system the charge does not accelerate. Therefore, the Lienard-Wiechert fields (5), (6) show that it does not radiate and its fields are bound fields. The electromagnetic fields of this charge are

$$
\boldsymbol{E} \neq 0 ; \quad \boldsymbol{B}=0 .
$$

The null magnetic field of (15) and the Poynting vector (7) prove that these fields have no linear momentum density. Hence, if a particle is associated with bound fields then it is not a real photon. Indeed, it is shown above that in any inertial frame, a real photon has a non-vanishing linear momentum.

\subsection{The Fields' Energy-Momentum Tensor}

The energy-momentum tensor of electromagnetic fields is a very important element of the theoretical structure of electrodynamics. Indeed, it is used in a 
relativistic proof of local conservation of energy-momentum and shows energymomentum currents throughout the entire space-time (see [1], pp. 601-608; [3], pp. 86-89). The energy-momentum tensor is a second rank tensor denoted by $T^{\mu v}$. Physical requirements, like a consistent definition of angular momentum as well as the laws of general relativity show that this tensor must be symmetric. Angular momentum arguments can be found in [1], pp. 601-608 and in [3], pp. 82-89. The energy-momentum tensor is also used in Einstein equations of general relativity (see [3], p. 297)

$$
R_{\mu v}-\frac{1}{2} g_{\mu v} R=8 \pi k T_{\mu \nu}
$$

where $R_{\mu v}$ is the second rank curvature tensor, $g_{\mu v}$ is the metric tensor and $k$ is the gravitational constant. The tensors $R_{\mu v}$ and $g_{\mu v}$ are symmetric. Therefore, general relativity requires a symmetric energy momentum tensor.

The variational principle is used for a derivation of the energy-momentum tensor of electromagnetic fields. It turns out that a direct derivation of this tensor yields the following asymmetric expression (see [3], p. 86)

$$
T^{\mu \nu}=-\frac{1}{4 \pi} \frac{\partial A_{\lambda}}{\partial x_{\mu}} F^{\nu \lambda}+\frac{1}{16 \pi} g^{\mu v} F_{\alpha \beta} F^{\alpha \beta} .
$$

The first term of (17) is an asymmetric second rank tensor whereas the second term is symmetric. Hence, the tensor $T^{\mu v}$ of (17) is inconsistent with the required symmetry of the energy-momentum tensor. Furthermore, there are two other objections to (17) (see [1], p. 604).

- Maxwell equations are gauge invariant. This property means that the energy-momentum tensor must depend only on the fields tensor $F^{\mu v}$.

- This tensor is not traceless, which means that it is inconsistent with the fact that the photon has no rest frame.

As stated in the introduction to this work, the variational principle is regarded as an important principle of physics. Its application yields (17), and the first term of this expression proves that an unacceptable result is obtained. This issue casts certain doubts concerning the merits of the variational principle as a fundamental element of theoretical physics [12]. Textbooks show how (17) can be corrected (see [3], pp. 86, 87). Here one adds to (17) the following term

$$
W^{\mu \nu}=\frac{1}{4 \pi} \frac{\partial A^{\mu}}{\partial x^{\lambda}} F^{v} \lambda=\frac{1}{4 \pi} \frac{\partial}{\partial x^{\lambda}}\left(A^{\mu} F^{v \lambda}\right) .
$$

Arguments claim that this operation is justified because it is a divergenceless quantity $W_{, v}^{\mu \nu}=0$, which is consistent with conservation laws. This operation settles all problems: It yields the well known symmetric traceless tensor which depends only on the electromagnetic fields (see [3], p. 87; [1], p. 605)

$$
T^{\mu v}=\frac{1}{4 \pi}\left(g^{\mu \alpha} F_{\alpha \beta} F^{\beta v}+\frac{1}{4} g^{\mu v} F_{\alpha \beta} F^{\alpha \beta}\right) \text {. }
$$

Although the above mentioned symmetrization procedure yields nice results, it has no profound theoretical basis. Indeed, if an addition of a divergenceless 
term to the energy-momentum tensor is a theoretically legitimate procedure then instead of adding the tensor $W^{\mu v}$ to (17), one may add the tensor $a W^{\mu v}$, where $a \neq 1$ is a real number. The unacceptable result obtained in this case means that an addition of a divergenceless tensor lacks the general validity which is required from a physically legitimate mathematical procedure.

Let us turn to the main objective of the present work-the examination of differences between radiation fields and bound fields. The following article [13] proves that radiation fields directly yield the symmetric tensor (19), because in this case the correction term $W^{\mu v}$ of (19) vanishes identically. This result means that the unphysical properties of the energy-momentum tensor which are derived from the electromagnetic fields Lagrangian density are related to bound fields only, whereas radiation fields directly yield the correct energy-momentum tensor.

\subsection{The Darwin Lagrangian}

As stated in the introduction to this work, the variational principle is used as a fundamental element of the theoretical structure of electrodynamics. Let us take a system of charges and examine their interaction with the fields of each other.

Maxwell equations prove that the fields depend on retarded quantities of each charge. Let $R$ denote the distance from the spatial point where the fields are calculated to the retarded position of a given charge (see [3], p. 174). Calculations show that electromagnetic potentials depend on the retarded position and on the retarded time of the charge. If the origin of the space-time coordinates is chosen at the point where the fields are calculated then the retarded quantities $(t, R)$ of a given charge are obtained from the following equation (see [3], p. 174)

$$
t+R(t) / c=0 \text {. }
$$

Here the speed of light $c$ is written explicitly. It is shown in (5) and (6) that the electromagnetic fields can be written as a sum of two terms. One term depends on the charge's velocity and the other term depends also on its acceleration (see [3], p. 175; [1], p. 657). At a large distance from the system of charges, velocity fields decrease like $R^{-2}$ whereas acceleration fields decrease like $R^{-1}$. The Poynting vector (7) which depends quadratically on the fields, represents energy current. Hence, velocity fields are related to bound fields and acceleration fields are related to radiation fields.

A power series expansion proves that velocity fields of a given charge can be replaced by an expression that depends on its position and its velocity at the instant when the fields are measured. This expression is called the Darwin Lagrangian (see [1], pp. 593-595; [3], pp. 179-182). The electromagnetic interaction terms of this Lagrangian take a mechanical-like form which depends on the instantaneous position and velocity of the charged particles

$$
L_{\text {Darwin,int }}=-\sum_{j} \sum_{i>j} \frac{e_{j} e_{i}}{R_{i j}}+\sum_{j} \sum_{i>j} \frac{e_{j} e_{i}}{2 R_{i j}}\left[\boldsymbol{v}_{j} \cdot \boldsymbol{v}_{i}+\left(\boldsymbol{v}_{j} \cdot \boldsymbol{n}_{i j}\right)\left(\boldsymbol{v}_{i} \cdot \boldsymbol{n}_{i j}\right)\right] .
$$

Here $\boldsymbol{v}_{i}, e_{i}$ denote respectively the velocity and charge of the ith particle and 
$j, i$ run on the particles' index. $R_{i j}$ is the distance between the ith and the jth particle and $\boldsymbol{n}_{i j}$ denotes the unit vector in the direction from the ith particle to the jth particle. The Darwin Lagrangian depends on the instantaneous value of the coordinates and of the velocity of the charged particles but is independent of the electromagnetic fields and of their potentials. In the quantum domain, the Darwin Lagrangian of a system of Dirac particles yields the Breit interaction (see [14], pp. 170, 195).

The next term in the power series expansion depends on the second derivative of the charge's coordinates with respect to time, namely, on its acceleration. The following article [15] proves that this term cannot be removed from the Lagrangian.

The Lagrangian function determines the dynamics of the system. It is shown above that velocity fields can be removed from the Lagrangian and their effect is replaced by appropriate terms of the Darwin Lagrangian (see [3], pp. 179-182). In the quantum domain, the Breit interaction takes the task of the Darwin Lagrangian (see [14], pp. 170, 195). By contrast, a mathematical analysis of the laws of Maxwellian electrodynamics proves that acceleration fields cannot be replaced by an appropriate mechanical-like term of the Lagrangian [15]. This result means that acceleration fields are related to a genuine entity. In classical physics it is the energy radiated by a given system and in the quantum domain it is called a real photon. The removal of velocity fields from the Lagrangian function means that they do not represent a genuine physical particle. This issue demonstrates another inherent difference between bound fields and radiation fields.

\subsection{Magnetic Monopoles}

It is recognized that a magnetic monopole (called briefly monopole) has not been detected in experiment. The monopole part of [7] presents a very long list of reports on this kind of experimental failure. For example, the CERN's ATLAS collaboration reports the failure of its recent experimental attempt aiming to detect monopoles: "No events were observed in data in the signal region" [16]. It is interesting to point out that this systematic failure of the monopole quest has been predicted a quite long time ago [17]. This state of affairs means that the monopole issue should be discussed theoretically. Evidently, this approach must rely on well established theoretical elements.

The following items outline very briefly how the required Regular ChargeMonopole Theory (RCMT) is constructed. Furthermore, they also show the obvious constraints that are imposed on this theory, and references to articles that explain appropriate details. Here the symbol $g$ is the monopole analog of the electric charge $e$.

1) As explained in the introduction section, also the RCMT should be constructed on the basis of the variational principle. Therefore, the following items explain how the Lagrangian of a classical monopole theory is constructed. 
Evidently, like in the case of electrodynamics of charged particles, this Lagrangian can be utilized for the construction of a quantum theory of monopoles.

2) The main objective is to find how monopoles are embedded in Maxwellian electrodynamics. The present form of the Lagrangian density of Maxwellian electrodynamics (2) can be regarded as the limit $g=0$ of the required Lagrangian function of the RCMT, because this limit holds for the standard form of Maxwellian electrodynamics, where $g=0$ throughout the entire space. Therefore, this limit is a constraint which is imposed on the required RCMT.

3) The next step is to find a theoretical definition of a monopole. This matter can be found in the standard literature, which utilizes the following duality transformation (see [18], p. 1363)

$$
E \rightarrow B, \quad B \rightarrow-E
$$

together with this charge transformation

$$
e \rightarrow g ; g \rightarrow-e .
$$

The fields transformations (22) can be put in a tensorial form

$$
F^{\mu \nu} \rightarrow F^{* \mu \nu} ; F^{* \mu \nu} \rightarrow-F^{\mu \nu}
$$

where $F^{* \mu v}=\epsilon^{\mu v \alpha \beta} F_{\alpha \beta}$. The transformations (22) and (23) are sometimes called duality rotations by $\pi / 2$ (see [1], p. 252).

4) An application of the duality transformations (22) and (23) to the Lagrangian density of electromagnetic fields and their interaction with charged particles (2), yield the following Lagrangian density of a monopole system

$$
\mathcal{L}_{\text {Monopole }}=-\frac{1}{16 \pi} F^{* \mu v} F_{\mu v}^{*}-g j^{\mu} G_{\mu} .
$$

Here $G_{\mu}$ is a sum of 4-potentials of monopoles, where each of which corresponds to the ordinary Lienard-Wiechert 4-potential of an electric charge. Note also that $F^{\mu v} F_{\mu v}=F^{* \mu v} F_{\mu v}^{*}$.

5) The foregoing application of the duality transformation (23) to a system of charges without monopoles yields (25), which is a system of monopoles without charges. The next assignment is to find the form of a Lagrangian density of a combined system of charges and monopoles. The previous point means that now we have a second constraint that is imposed on the RCMT: In the case of a chargeless system, the charge-monopole Lagrangian density should take the form of (25). This constraint is dual to that of item 2 above.

6) Relying on mathematical properties of Maxwellian electrodynamics, it is proved that a regular Lagrangian function can be constructed for the fields of a charge-monopole system, provided bound fields of charges, bound fields of monopoles and radiation fields are treated separately [19] [20]. This separation between the fields is compatible with the linearity of Maxwell equations. It should be noted that the analysis assumes nothing on the specific properties of these fields. It means that the results are derived mathematically. The Lagrangian function which is obtained in this way shows that bound fields and radiation 
fields have different dynamical properties. This result can be regarded as an additional proof of the main assertion of the present work: bound fields and radiation fields have different physical properties.

The Lorentz invariant (9) takes a negative value for the bound field of a charge; it takes a positive value for the bound field of a monopole; it takes a null value for radiation fields emitted from a system of charges and for radiation fields emitted from a system of monopoles. The distinction between these three kinds of fields, which is used in the RCMT construction, is consistent with these relativistic properties.

The monopole theory that is described above is used as an element of the long lasting successful prediction of the failure of the present form of experimental monopole search [17]. This evidence indicates the physical merits of the monopole theory described above.

\section{Discussion}

In principle, one solid support of an inherent difference between two physical objects justifies its acceptance. Therefore, the above mentioned wide variety of distinct arguments, where each of which shows inherent physical differences between radiation fields (namely, real photons) and bound fields, provide a reliable basis for the main assertion of this work: Radiation fields and bound fields do not represent the same physical object. The following lines point out few examples showing that this conclusion solves some theoretical problems and also explains experimental effects that are unsettled in the present mainstream literature.

It is shown in subsection 3.7 that a symmetric energy momentum tensor is required for a consistent definition of the fields angular momentum (see [3], p. 84). Moreover, (17) proves that in the present literature the Lagrangian density of electromagnetic fields does not yield a symmetric energy-momentum tensor. This is certainly a problem because the photon's helicity is a well defined quantity, and this property indicates that a photon has a well defined angular momentum $j=1$ (see the photon data in [7]). Therefore, the symmetric energy momentum tensor that is directly obtained from the radiation fields Lagrangian density and the interrelations between classical and quantum electrodynamics indicate that if radiation fields and bound fields are treated separately then the photon's angular momentum is defined consistently.

The analysis of subsection 3.8 shows how electromagnetic velocity fields of the classical Lagrangian are removed and the mechanical-like Darwin Lagrangian is derived. This Lagrangian depends on the instantaneous position and velocity of the charged particles. Furthermore, the Breit interaction represents the effects of the Darwin Lagrangian in the quantum domain. An analogous feature is shown in subsection 3.3 which discusses angular momentum and parity of an atomic quantum state. Here the electromagnetic bound fields of the atomic charged constituents are ignored, and the wave function of the massive constituents of 
the atom yields eigenvalues of the system's angular momentum and parity.

The calculation of the 4-potential (4) and its fields (5) and (6) depends on retarded quantities. This issue indicates that these expressions are related to a genuine physical object which cannot travel faster than light. An indeed, (5) and (6) contain expressions for radiation fields, which are related to a genuine quantum object which is called a photon. The introduction of the Darwin Lagrangian and the associated Breit interaction bears a profound change in the interpretation of bound fields. The instantaneous quantities which are used in the Darwin Lagrangian and in the Breit interaction mean that bound fields cannot represent a genuine physical object simply because a genuine physical object cannot travel faster than light.

The primary assertion of this work says that radiation fields and bound fields do not represent the same physical object. The topics that are mentioned in the previous two paragraphs go one step further. By showing that important physical quantities like energy, angular momentum and parity are derived only from the electronic wave function, they indicate that bound fields do not represent a genuine independent physical object but are just auxiliary mathematical quantities.

Another issue pertains to the theoretical structure of quantum electrodynamics (QED) which uses the following form of the Lagrangian density of free electromagnetic fields (see [4], p. 342; [21], p. 70)

$$
\mathcal{L}_{E M}=-\frac{1}{16 \pi} F^{\mu v} F_{\mu v} .
$$

Here the fields tensor $F^{\mu v}$ is a sum of bound fields and radiation fields

$$
F^{\mu v}=F_{(B o u)}^{\mu v}+F_{(R a d)}^{\mu v} \text {. }
$$

A substitution of (27) into (26) proves that the present structure of the QED Lagrangian density of free electromagnetic fields is

$$
\mathcal{L}_{E M}=-\frac{1}{16 \pi}\left[F_{(B o u)}^{\mu v} F_{(B o u) \mu \nu}+F_{(\text {Rad })}^{\mu v} F_{(\text {Rad }) \mu v}+2 F_{(\text {Bou })}^{\mu v} F_{(\text {Rad }) \mu v}\right] .
$$

The last term of (28) means that this expression is unacceptable. Indeed, radiation fields represent a photon whose parity is negative (see the photon data in [7]), whereas the discussion of the Zeeman effect which is presented in subsection 3.1 proves that a magnetic bound field has an even parity. Furthermore, the conclusions of subsection 3.3 show that atomic bound fields must have an even parity. Let us use these issues and see how a parity transformation affects (28) in the case of the static magnetic field which is used in the Zeeman effect of subsection 3.1. The even parity of $F_{(\text {Bou })}^{\mu v}$ and the odd parity of $F_{(R a d)}^{\mu v}$ prove that the first and the second terms of (28) do not change sign: $(+1)^{2}=1$ and $(-1)^{2}=1$. On the other hand, the even parity of the magnetic field and the odd parity of the photon prove that the last term of (28) changes sign. Thus, a parity transformation of the Lagrangian density (28) yields

$$
\mathcal{L}_{E_{E} \_P}=-\frac{1}{16 \pi}\left[F_{(B o u)}^{\mu v} F_{(B o u) \mu v}+F_{(R a d)}^{\mu v} F_{(R a d) \mu v}-2 F_{(B o u)}^{\mu v} F_{(R a d) \mu v}\right] .
$$


Hence, the last term of (28) violates parity, whereas electrodynamics conserves it. Angular momentum arguments yield an analogous result.

This theoretical QED contradiction is supported by the following experimental evidence: QED calculations of electron data and of muon data of the proton charge radius yield inconsistent results (see [22] and the proton data of [7]). This evidence means that QED has already lost its long-lasting reputation as an extremely accurate theory.

The last example refers to the RCMT of subsection 3.9. One result that is derived from this theory says that the dynamics of this system, which consists of electric charges and magnetic monopoles, abide by the following laws:

A. Charges do not interact with bound fields of monopoles; monopoles do not interact with bound fields of charges; radiation fields of the system are identical and charges as well as monopoles interact with them.

Furthermore, another result of the RCMT says that the size of an elementary monopole $g$ is a free parameter and like the electric charge, it should be derived from experiments.

Conclusion A is obtained from a pure theoretical analysis [19] [20]. It turns out that it has a striking manifestation in physical data. Indeed, real photons represent electromagnetic radiation; electrons represent electric charge and quarks represent dyons, which are particles that carry an electric charge and a magnetic monopole [23]. Experiment proves that in quarks $g \gg e$. This is the reason that explains why strong interactions are much stronger than electromagnetic interactions. Moreover, the data prove the following evidence:

B. Electrons do not interact with strong fields of quarks (see [24], p. 2); quarks interact electromagnetically with bound fields of electrons (see the data on deep inelastic electron-quark scattering, [25], pp. 266-274); photons interact with electrons as well as with the strong monopole charge of quarks [26]. The fact that $g \gg e$ and the last point explain why the hard photon interaction with a proton is very similar to the corresponding interaction with a neutron.

The impressive correspondence between the elements of item A, which are derived from theoretical considerations, and those of item $B$, which describe a collection of experimental data, provide a strong support for the validity of the RCMT.

It is interesting to note that several very well known authors have already put forward the idea that monopoles are constituents of hadrons (see e.g. [27] [28] [29] [30]). The RCMT and, in particular, the correspondence between the elements of the theoretical item $\mathrm{A}$ and the corresponding elements of the experimental item B, provide an important support for this idea. Many other aspects of the correspondence between the RCMT and strong interactions are discussed in [31] [32] [33].

An important point of the forgoing discussion is put in the following statements. Experimental data prove the following properties of the two primary QED particles: Electrons do not participate in strong interactions (see [24], p. 2). 
By contrast, a real photon participates in strong interactions [26]. Evidently, a real photon is a very important elementary particle. Referring to the strong interactions of a real photon with hadrons, it is quite strange to realize that today it is very hard to find an appropriate discussion of this topic in modern QFT textbooks as well as in particle physics textbooks. The present work may contribute to attempts aiming to close this gap.

\section{Conclusions}

This work discusses properties of radiation fields and bound fields of electrodynamics. The variational principle is used as a basis for the theoretical discussion. Some examples belong to classical physics and other examples belong to the quantum domain. The main result of this work proves that radiation fields and bound fields represent inherently different physical objects. The wide variety of different cases, which are described in Section 3, provide a good basis for the main assertion of this work. It is proved in the penultimate section that the QED Lagrangian density is affected by the outcome of this work. It is also explained why the results of this work together with the variational principle indicate that magnetic monopoles are linked to strong interactions.

It is interesting to mention that a discussion based on the main assertion of this work solves the QED problems of infinite physical quantities [34]. At present, these infinities are removed by a process called renormalization. Renormalization is certainly a doubtful process because it takes the mathematically unacceptable difference between two infinite quantities. One should realize that two eminent QED figures have used the following words for denying renormalization: Dirac said that it has an "illogical character" [35] whereas Feynman called it "a dippy process" [36]. An analogous opinion is put forward in Ryder's QFT textbook (see [37], p. 390). This author compares quantum divergences to classical ones and says: "In the quantum theory, these divergences do not disappear; on the contrary, they appear to get worse, and despite the comparative success of renormalisation theory the feeling remains that there ought to be a more satisfactory way of doing things." As mentioned in the previous section, the failure of QED to explain the proton charge radius data supports the above mentioned theoretical arguments concerning the problematic status of the present form of QED.

\section{References}

[1] Jackson, J.D. (1975) Classical Electrodynamics. John Wiley, New York.

[2] https://en.wikipedia.org/wiki/Heinrich_Hertz

[3] Landau, L.D. and Lifshitz, E.M. (2005) The Classical Theory of Fields. Elsevier, Amsterdam.

[4] Weinberg, S. (1995) The Quantum Theory of Fields, Vol. I. Cambridge University Press, Cambridge.

[5] Griffiths, D. (2008) Introduction to Elementary Particles. 2nd Edition, Wiley-VCH, 
Weinheim.

[6] Schiff, L.I. (1955) Quantum Mechanics. McGraw-Hill, New York.

[7] Patrignani, C., et al. (Particle Data Group) (2016) The Review of Particle Physics. Chinese Physics, 40, 100001. (2017 update) http://pdg.lbl.gov/

[8] Landau, L.D. and Lifshitz, E.M. (1959) Quantum Mechanics. Pergamon, London.

[9] Wigner, E. (1939) On Unitary Representations of the Inhomogeneous Lorentz Group. Annals of Mathematics, 40, 149-204. https://doi.org/10.2307/1968551

[10] Schweber, S.S. (1964) An Introduction to Relativistic Quantum Field Theory. Harper \& Row, New York, 44-53.

[11] Sternberg, S. (1994) Group Theory and Physics. Cambridge University Press, Cambridge, 143-150.

[12] Munoz, G. (1996) Lagrangian Field Theories and Energy-Momentum Tensors. American Journal of Physics, 64, 1153-1157. https://doi.org/10.1119/1.18336

[13] Comay, E. (2018) A Consistent Construction of the Electromagnetic Energy-Momentum Tensor. Open Access Library Journal, 5, e4354.

https://www.scirp.org/journal/PaperInformation.aspx?PaperID=82391 https://doi.org/10.4236/oalib.1104354

[14] Bethe, H.A. and Salpeter, E.E. (1957) Quantum Mechanics of One- and Two-Electron Atoms. Springer, Berlin.

[15] Comay, E. (1991) Electromagnetic Energy-Momentum Tensor and Elementary Classical Point Charges. International Journal of Theoretical Physics, 30, 1473-1487. https://doi.org/10.1007/BF00675612

[16] Aad, G., et al. (2016) Search for Magnetic Monopoles and Stable Particles with High Electric Charges in $8 \mathrm{TeV} p p$ Collisions with the ATLAS Detector. Physical Review $D$, 93, Article ID: 052009. https://doi.org/10.1103/PhysRevD.93.052009

[17] Comay, E. (1985) Will Magnetic Monopoles Be Detected in Our Instruments? Lettere al Nuovo Cimento, 43, 150-152. https://doi.org/10.1007/BF02749596

[18] Goddard, P. and Olive, D.I. (1978) Magnetic Monopoles in Gauge Field Theories. Reports on Progress in Physics, 41, 1357-1437. https://doi.org/10.1088/0034-4885/41/9/001

[19] Comay, E. (1984) Axiomatic Deduction of Equations of Motion in Classical Electrodynamics. Il Nuovo Cimento B, 80, 159-168. https://doi.org/10.1007/BF02722256

[20] Comay, E. (1995) Charges, Monopoles and Duality Relations. Il Nuovo Cimento B, 110, 1347-1356. https://doi.org/10.1007/BF02723118

[21] Bjorken, J.D. and Drell, S.D. (1965) Relativistic Quantum Fields. McGraw-Hill, New York.

[22] Pohl, R., et al. (2010) The Size of the Proton. Nature, 466, 213-216. https://doi.org/10.1038/nature09250

[23] https://en.wikipedia.org/wiki/Dyon

[24] Halzen, F. and Martin, A.D. (1984) Quarks and Leptons: An Introductory Course in Modern Particle Physics. John Wiley, New York.

[25] Perkins, D.H. (1987) Introduction to High Energy Physics. Addison-Wesley, Menlo Park.

[26] Bauer, T.H., Spital, R.D., Yennie, D.R. and Pipkin, F.M. (1978) The Hadronic Properties of the Photon in High-Energy Interactions. Reviews of Modern Physics, 50, 261. https://doi.org/10.1103/RevModPhys.50.261 
[27] Dirac, P.A.M. (1948) The Theory of Magnetic Poles. Physical Review Letters, 74, 817-830. https://doi.org/10.1103/PhysRev.74.817

[28] Schwinger, J. (1968) Sources and Magnetic Charge. Physical Review, 173, 1536. https://doi.org/10.1103/PhysRev.173.1536

[29] Schwinger, J. (1969) A Magnetic Model of Matter. Science, 165, 757-761. https://doi.org/10.1126/science.165.3895.757

[30] Barut, A.O. (1971) Proton Form-Factor, Magnetic Charges, and Dyonium. Physical Review, D3, 1747-1750. https://doi.org/10.1103/PhysRevD.3.1747

[31] Comay, E. (2004) A Regular Theory of Magnetic Monopoles and Its Implications. In: Chubykalo, A., Onoochin, V., Espinoza, A. and Smirnov-Rueda, R., Eds., Has the Last Word Been Said on Classical Electrodynamics? Rinton Press, Paramus, 335.

[32] Comay, E. (2012) The Regular Charge-Monopole Theory and Strong Interactions. Electronic Journal of Theoretical Physics, 9, 93-118.

http://www.ejtp.com/articles/ejtpv9i26p93.pdf

[33] Comay, O. (2014) Science or Fiction? The Phony Side of Particle Physics. S. Wachtman's Sons.

[34] Comay, E. (2015) Interrelations between Mathematics and Experiment in the Present Structure of Quantum Electrodynamics. OALibJ, 2, 1-6.

https://www.scirp.org/journal/PaperInformation.aspx?PaperID=69004

[35] Dirac, P.A.M. (1963) The Evolution of the Physicist's Picture of Nature. Scientific American, 208, 45-53. https://doi.org/10.1038/scientificamerican0563-45

[36] Feynman, R.P. (1990) QED, the Strange Theory of Light and Matter. Penguin, London.

[37] Ryder, L.H. (1997) Quantum Field Theory. Cambridge University Press, Cambridge. 